\title{
Radical Resection of Adult Low Grade Oligodendroglioma without Adjuvant Therapy: Results of a Prospective Treatment Protocol
}

\author{
—Surgical Treatment of Low-Grade Oligodendroglioma
}

\author{
Donald A. Ross ${ }^{1 *}$, Lynda Yang ${ }^{2}$, Oren Sagher ${ }^{2}$, Amy M. Ross ${ }^{3}$ \\ ${ }^{1}$ Department of Neurological Surgery, Oregon Health and Science University, Portland, USA; ${ }^{2}$ Department of Neurological Surgery, \\ University of Michigan, Ann Arbor, USA; ${ }^{3}$ School of Nursing, Oregon Health and Science University, Portland, USA. \\ Email: drdoom867@yahoo.com
}

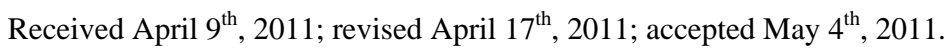

\begin{abstract}
The goal of this work was to demonstrate prospectively that maximal surgical resection of low grade oligodendrogliomas without adjuvant therapy does not reduce life expectancy over that of historical controls. All patients with surgically accessible grade II oligodendrogliomas underwent maximal resection using stereotactic guidance and/or cortical mapping and were followed with serial MRI scans without adjuvant therapy until either progression or spread into brain regions deemed not surgically resectable. Nineteen patients were treated between 1993 and 2006. Ten patients required reoperation an average of 55 months after their first surgery. Nine patients progressed to anaplastic tumors an average of 42 months after their first surgery: six patients died from their tumors an average of 73 months after diagnosis, two are still alive 76 and 18 months after progression, and one was lost to follow up. Ten patients are alive and progression-free an average of 116 months after diagnosis, one of whom was lost to follow up at 106 months from diagnosis. Four patients are alive and event-free an average of 125 months after diagnosis. All are male and three had tumors in the superior frontal gyrus. The event-free survival, progression-free survival, and overall survival of our patients are not worse than those of patients treated with postoperative adjuvant therapy. Withholding adjuvant therapy at diagnosis appears to be safe. It will be important to establish the molecular differences between the patients who did very well and those who progressed so that adjuvant therapy could be offered to the latter.
\end{abstract}

Keywords: Corticography, Craniotomy, Extent of Resection, Oligodendroglioma

\section{Introduction}

The management of low-grade oligodendrogliomas has been controversial, without a consensus in the neurooncological community on the role and timing of surgery [1,2] and of adjuvant radiation and chemotherapy [1,3-7]. For 19 years, the senior author [DAR] has prospectively managed low grade oligodendrogliomas amenable to surgery with radical resection using stereotactic guidance and intraoperative cortical mapping when indicated, followed by observation without adjuvant therapy until histological progression or extension into unresectable areas. This protocol was based, in part, in the belief that radical cytoreduction might reduce the risk of malignant transformation [8], the ultimate cause of death of most patients harboring low-grade glial neoplasms [9]. The results of this management protocol are reported.

\section{Methods}

In 1990, a prospective management protocol for low grade [WHO Grade II] oligodendrogliomas was begun which recommended radical resection followed by observation with serial MRI and the withholding of adjuvant therapy until demonstrated histopathological progression to a higher grade tumor or until the tumor spread into areas of the brain which contraindicated further resection. Tumors thought suitable for this protocol were generally areas of T1 shortening and T2 signal increase [prolongation] on MRI without gadolinium enhancement. Tumors not amenable to radical resection were biopsied stereotactically and then either observed or treated adjuvantly, and were not included in this study. Resections were always carried out with stereotactic 
guidance, initially frame based [Leksell, Elekta], and later, frameless [Stealth, Medtronic]. When tumors were immediately adjacent to or potentially within eloquent cortical or subcortical structures, surgery was carried out under local anesthesia with bipolar corticography and intraoperative EEG. Extent of resection was quantified by postoperative MRI obtained within 48 hours of surgery. Pathology of grade II oligodendroglioma by a neuropathologist using WHO criteria [10] resulted in withholding of adjuvant therapy and the initiation of regular clinical and MRI follow up at 6 month intervals. All patients underwent baseline MRI 2-3 months postoperatively to allow surgical edema to subside. This scan was used as the baseline for future scans. Tumor recurrences judged to be resectable were reoperated according to the same protocol. If the tumor grade had not changed, then no adjuvant therapy was given. If tumors progressed to a higher grade or invaded eloquent structures, then adjuvant therapy was recommended. Institutional Review Board approval for this study was obtained both at the University of Michigan Hospitals and at Rogue Valley Medical Center.

\section{Results}

Patients in this study were operated upon between 1993 and 2006. During that time, the senior author operated upon 877 intracranial tumors, of which 318 were gliomas. Nineteen patients with pure, grade II oligodendrogliomas were treated (Table 1). Eleven presented with a seizure. Eleven patients were male. Ages ranged from 20 to 71, average 38 years. Only three patients were older than 50 years. Ten tumors were in the frontal lobe, six in the temporal lobe, two in the parietal lobe, and one in the occipital lobe. Eleven tumors were in the right hemisphere, and eight in the left. No patient sustained a new neurologic deficit or other serious complication as a result of surgery.

Complete follow up was available on 17 patients. Two were lost to follow up at 32 and 106 months postoperatively. Overall survival, event free survival, and progression free survival are shown in Figures 1-3 as Kaplan Meier plots. Ten patients required reoperation an average of 55 months after their first surgery. Nine patients progressed to anaplastic tumors an average of 42 months after their first surgery [exact time of progression was uncertain for three]: six patients died from their tumors an average of 73 months after diagnosis, two are still alive 76 and 18 months after progression, and one was lost to follow up. Ten patients are alive and progression-free an average of 116 months after diagnosis, one of whom was lost to follow up at 106 months from diagnosis. Four patients are alive and event-free an average of 125 months after diagnosis. All four are male and three had tumors in the superior frontal gyrus and one in the right temporal lobe. Two are surgeons and are working full time.

\section{Illustrative Cases}

Patient 1 was a 38 year-old male surgeon who had a single seizure. MRI showed a mass in the posterior aspect of the right superior frontal gyrus (Figures 4 and 5). The

Table 1. Patient characteristics.

\begin{tabular}{|c|c|c|c|c|c|}
\hline Age & Gender & $\begin{array}{l}\text { Surgery Date } \\
\text { (months) }\end{array}$ & $\begin{array}{l}\text { Time to Reop } \\
\text { (months) }\end{array}$ & $\begin{array}{l}\text { Prog Time } \\
\text { (months) }\end{array}$ & Survival \\
\hline 30 & $\mathrm{M}$ & $9 / 9 / 02$ & 49 & & 69 \\
\hline 43 & M & 9/8/99 & 25 & 84 & 102 \\
\hline 29 & M & 7/10/98 & 17 & 39 & 115 \\
\hline 36 & $\mathrm{~F}$ & $12 / 1 / 97$ & 9 & 13 & 52 (D) \\
\hline 43 & M & $11 / 5 / 93$ & & & 115 \\
\hline 34 & M & $12 / 1 / 99$ & & & 102 \\
\hline 26 & $\mathrm{M}$ & $12 / 16 / 94$ & & ? & 39 (D) \\
\hline 54 & $\mathrm{~F}$ & $5 / 19 / 95$ & & 32 & 32 (LTF) \\
\hline 39 & $\mathrm{~F}$ & $6 / 27 / 01$ & 60 & & 84 \\
\hline 29 & M & $2 / 2 / 96$ & & & 148 \\
\hline 38 & M & $10 / 29 / 97$ & & & 129 \\
\hline 29 & $\mathrm{~F}$ & $12 / 12 / 97$ & 76 & & 126 \\
\hline 71 & M & 8/11/99 & & ? & $53(\mathrm{D})$ \\
\hline 44 & $\mathrm{~F}$ & $12 / 12 / 97$ & & 46 & 67 (D) \\
\hline 54 & $\mathrm{~F}$ & $4 / 24 / 98$ & 50 & & 122 \\
\hline 39 & M & $5 / 8 / 95$ & 77 & ? & 85 (D) \\
\hline 25 & $\mathrm{~F}$ & 7/19/96 & & 34 & 128 (D) \\
\hline 20 & $\mathrm{~F}$ & $4 / 12 / 93$ & 73,106 & & 106 (LTF) \\
\hline 52 & M & 4/21/95 & 117 & & 158 \\
\hline \multicolumn{2}{|c|}{$\mathrm{D}=$ Died } & \multicolumn{4}{|c|}{ LTF= Lost to follow-up } \\
\hline
\end{tabular}




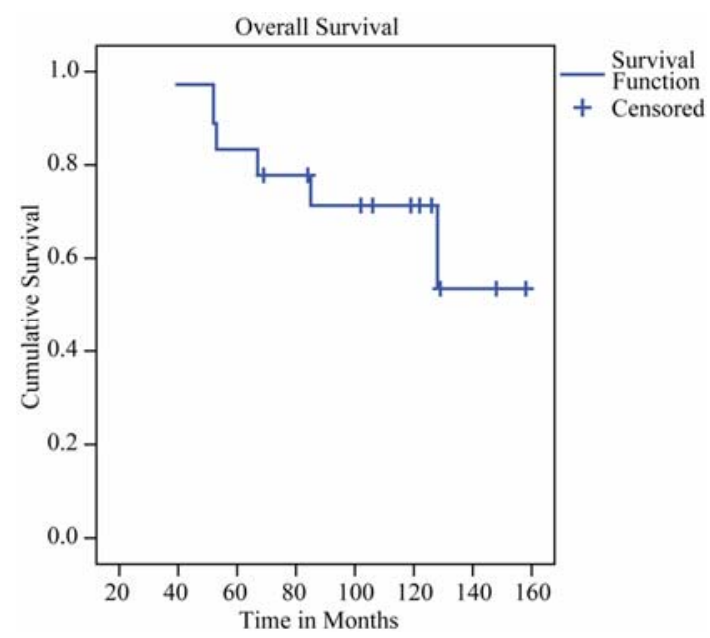

Figure 1. Kaplan-Meier plot of overall survival.

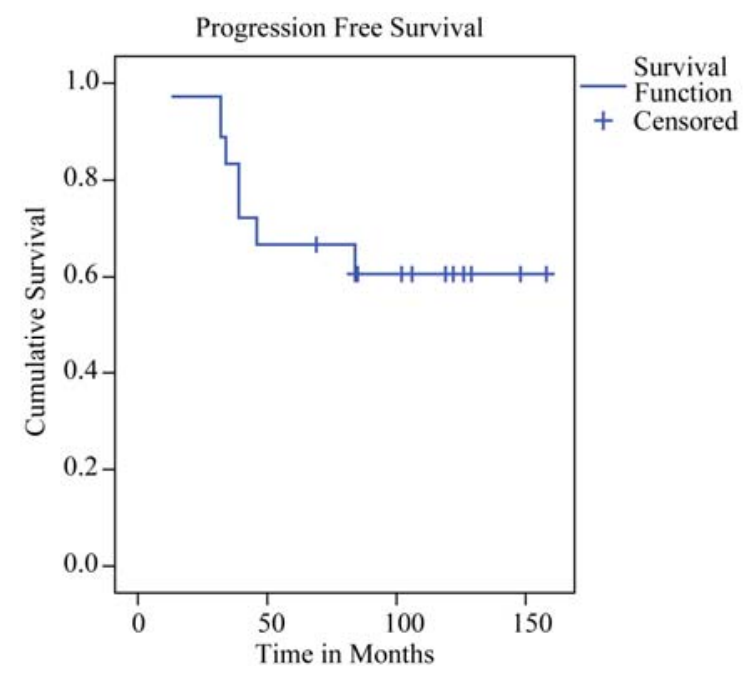

Figure 2. Kaplan-Meier plot of progression free survival.

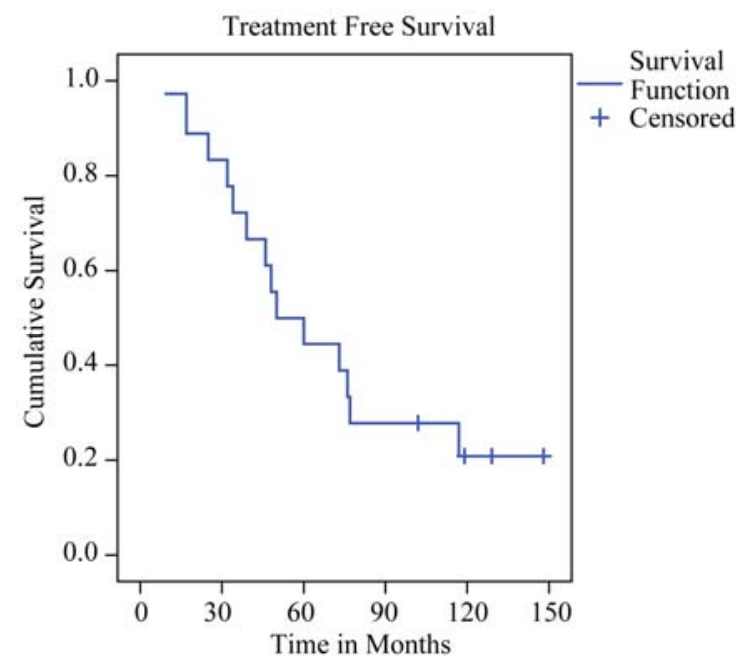

Figure 3. Kaplan-Meier plot of treatment free survival.

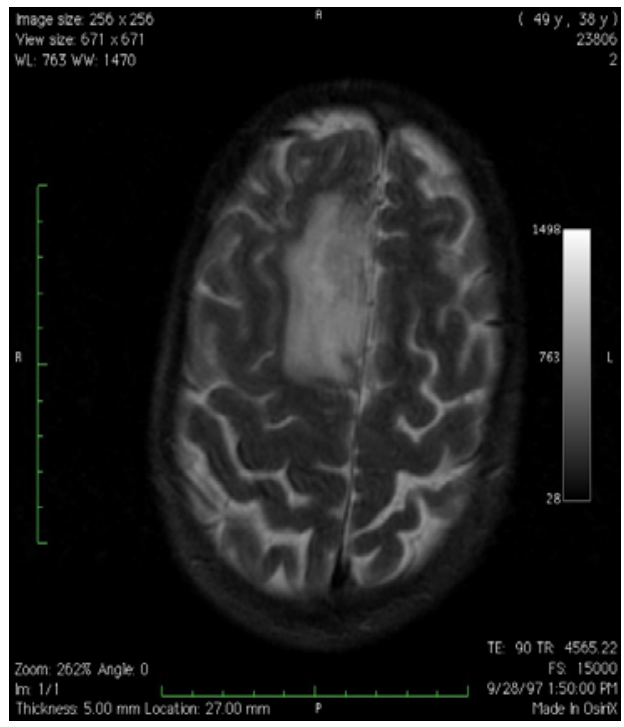

Figure 4. Axial T2-weighted MRI showing an area of T2 signal shortening in the right superior frontal gyrus which was a grade II oligodendroglioma at surgery.

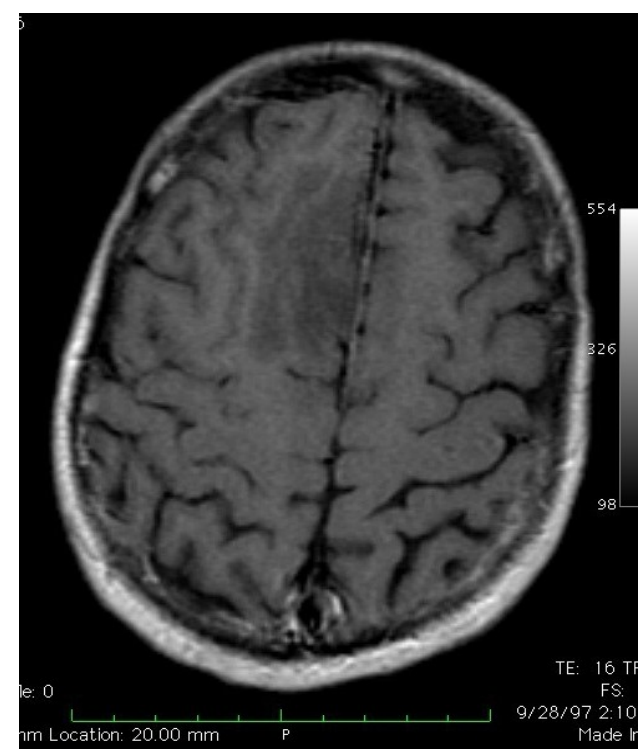

Figure 5. Axial T1-weighted gadolinium enhanced MRI showing an area of hypointensity in the right superior frontal gyrus without enhancement.

mass was radically resected at craniotomy under local anesthesia with stereotactic guidance and electrocortico graphy. No adjuvant therapy was given. The patient remains free of recurrence or progression 13 years postoperatively (Figure 6).

Patient 2 was a 44 year-old female with recurrent trigeminal neuralgia. An MRI obtained in evaluation of her neuralgia showed an incidental, asymptomatic mass in the tip of the right temporal lobe consistent with a low- 


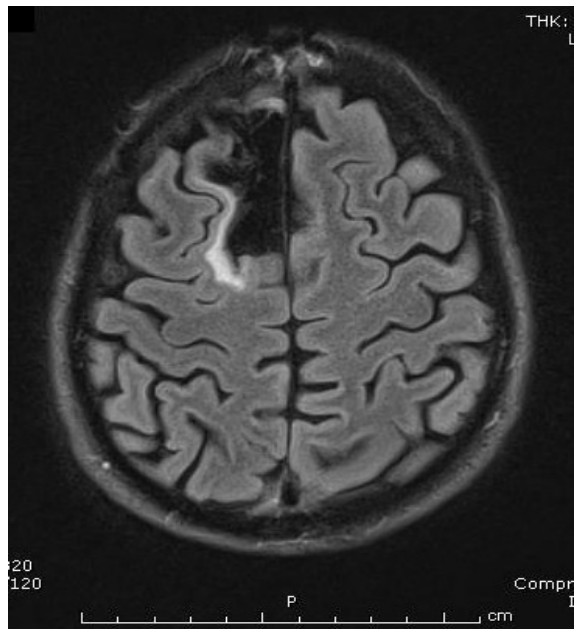

Figure 6. Axial FLAIR MRI obtained 128 months postoperatively.

grade glioma (Figure 7). The mass had not been present on previous MRIs obtained less than 2 years prior.

The mass was resected including a margin of 2 centimeters and was found to be a grade II oligodendroglioma. No adjuvant therapy was given. A recurrence 46 months later was called a glioblastoma, and was treated with adjuvant radiation and chemotherapy (Figure 8). The patient died 67 months after diagnosis.

\section{Discussion}

Potential reasons for withholding radiation therapy at diagnosis of WHO grade II oligodendroglioma include favorable natural history, benefit of gross total resection, lack of definitively demonstrated efficacy for radiation,

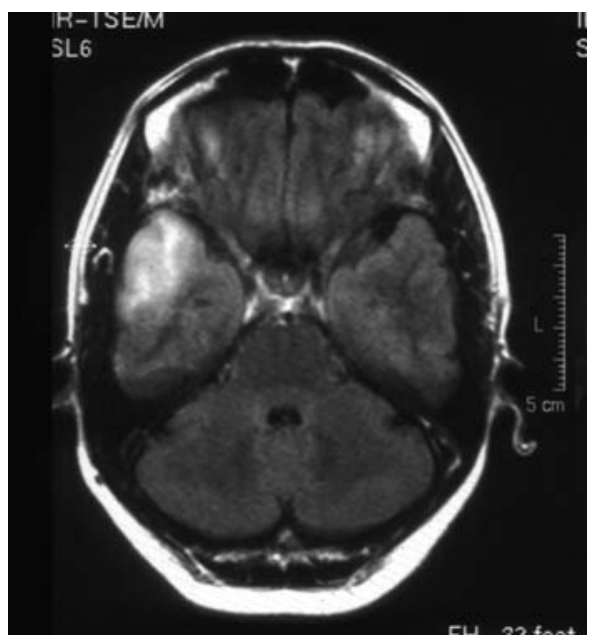

Figure 7. Axial, T2-weighted MRI scan showing an area of T2 signal shortening in the anterolateral aspect of the right temporal lobe which was a grade II oligodendroglioma at surgery.

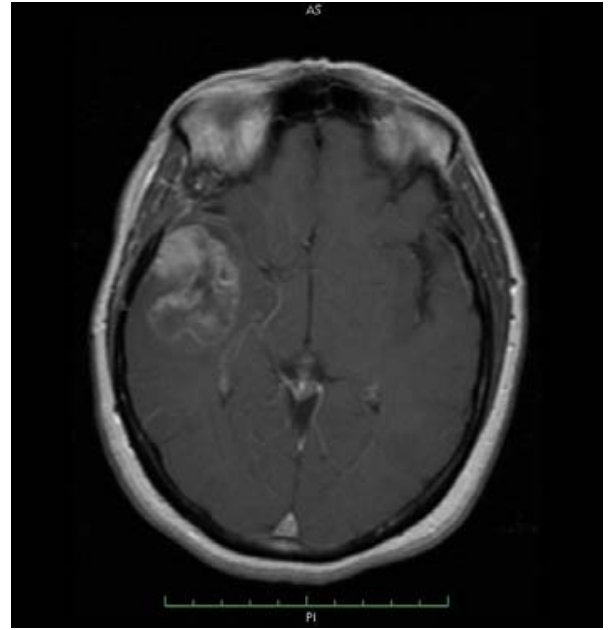

Figure 8. Axial, T1-weighted, gadolinium-enhanced MRI scan showing a glioblastoma in the right temporal lobe at the site of the previous oligodendroglioma.

potential for high-grade radiation toxicity, radiation induced cognitive dysfunction, or radiation related accelerated malignant progression, and the availability of effective chemotherapy.

Our data show that half of our patients are alive and progression free an average of 116 months after diagnosis without adjuvant therapy, consistent with previous reports of the favorable natural history of many oligodendrogliomas. Three patients are alive and event-free an average of 127 months after diagnosis. Yet, patients like Patient 1 above progressed and died despite early detection and aggressive surgery. This disparity of outcome indicates the heterogeneous nature of the tumors despite their common histologic features.

Although a pathological diagnosis of oligodendroglioma has traditionally depended upon light microscopic features, in recent years, the detection of losses at chromosome 1p36 and 19q13 have become more common determinants of oligodendroglial origin [11,12] and have been linked to better prognosis for response to adjuvant treatment [13-17]. A translocation may be responsible for many of these losses, may be linked to better overall survival, and may be most common in lesions found in the frontal lobes $[9,16]$. As more tumors are subjected to this analysis, it may be that the definition of an oligodendroglioma will narrow, and older series based on light microscopic definition may be found to contain tumors no longer thought to be oligodendrogliomas. This factor may account for some of the conflicting results from earlier studies, as noted below, and the variable outcomes of our patients.

The degree to which surgical resection influences survival remains controversial, and no prospective, random- 
ized trial has been conducted to answer this question [9, 18-20]. A retrospective study of 167 patients with various low-grade gliomas found that minimal postoperative residual tumor was associated with an improved 5-year survival rate [21]. Another retrospective study of 216 patients with various types of hemispheric low grade gliomas showed an improved outcome with greater extent of resection [20]. Keles [18] reviewed the literature and determined that a preponderance of the evidence favored resection over biopsy only. In analyzing the EORTC data, it was noted in the univariate analysis that greater than $90 \%$ resection was associated with longer overall survival, but the result was diminished greatly in the multivariate analysis [22]. In summarizing the results of multiple prospective trials involving almost 1000 patients [23], it was noted that extent of resection was a significant prognostic variable [along with age, histology, and tumor size]. In a stereotactic radiation study [24], extent of resection, was found not to be predictive of survival. A recent report on the role of intraoperative MRI in the resection of low-grade gliomas noted an increased risk of recurrence and death with subtotal resection [25]. Some studies suggest a reduced rate of malignant transformation with aggressive surgical resection [8, 26,27].

With respect to efficacy of postoperative radiation, some retrospective studies have suggested that there is benefit to postoperative radiotherapy for low-grade oligodendrogliomas [28-31], but prospective trials have appeared only recently. Two prospective, randomized, dose response trials reported no improvement with higher doses of radiation when compared to lower doses [32,33] and greater toxicity in the higher dose arm [33]. The lack of a dose response may be interpreted by some to indicate a lack of efficacy.

A prospective, 24 center, European trial of early versus delayed radiotherapy randomized 157 patients with various low-grade glioma histologies to early radiation [54Gy in $1.8 \mathrm{~Gy}$ fractions] and 157 patients to deferred radiation until time of progression [34]. Progression free survival was longer in the early radiation group, but overall survival was not significantly different. Among the 314 patients were 42 with oligodendrogliomas, of whom 19 were randomized to delayed radiation and 23 to early radiation. Outcomes for these patients were not reported separately from the entire group. The absence of a survival benefit to early irradiation was attributed to the efficacy of salvage radiotherapy at recurrence. The results of this trial have been disputed due to the inclusion of multiple tumor histologies, errors in pathologic grading, the lack of accurate determination of the extent of resection, its intention-to-treat paradigm [35], and for its lack of quality-of-life data [6].

In another prospective study, 18 patients were given no adjuvant treatment after gross total resection, or chemotherapy after subtotal resection or biopsy. At a median follow-up of 4.7 years, all patients were alive and only one had progressed (6\%) [36]. Fifteen patients had pure, low-grade oligodendrogliomas, but only five of the 18 underwent total resection by postoperative MRI. One patient who underwent gross total resection and no adjuvant therapy recurred at 2.5 years and underwent successful chemotherapy, but it is not clear if this patient had a pure oligodendroglioma or was one of the three with oligoastrocytomas.

When stereotactic radiation therapy was given at diagnosis for 24 WHO grade II oligodendrogliomas and 24 oligoastrocytomas, survival rates of $89 \%$ at 5 years and $74 \%$ at 10 years have been reported [24]. Progression-free survival was $52 \%$ at five years. It is not possible from this report to determine which patients had gross total resections. When compared to our data showing $50 \%$ progression free at greater than 9 years, there at least appears to be no harm in withholding adjuvant therapy.

In summary, multiple radiotherapy dose response trials have not demonstrated a dose response in low-grade oligodendroglioma, nor have prospective, randomized trials demonstrated a survival benefit for radiotherapy versus withholding radiation until progression.

Reasons for withholding adjuvant radiotherapy have included the risk of radiation induced cognitive dysfunction [34] or high grade radiation toxicity [33]. In a study involving 51 patients from a single institution, postoperative radiation therapy was associated with significantly worse cognitive function and more severe leukoencephalopathy on MRI [37]. However, a case control study failed to detect any evidence of cognitive decline when fractions of less than 2 Gray were used [38]. Radiation has also been thought to increase the risk of malignant transformation [9], but the recent European randomized trial did not find a higher incidence of malignant progression in the early radiation group [34]. In contrast, early radiation may improve seizure control in some patients [6,39]. Therefore, given the improved time to progression and improved seizure control with early radiotherapy, some have argued in favor of early treatment [6].

Since the recognition of the chemosensitivity of oligodendroglial tumors more than 15 years ago, there is now some tendency to use chemotherapy rather than radiation as the initial adjuvant therapy in oligodendrogliomas $[4,6,40]$.

The event-free survival, progression-free survival, and overall survival of our patients are not worse than those 
of patients treated with postoperative adjuvant therapy. Strict comparison with previous series is difficult because many contain several types of low-grade gliomas rather than only oligodendrogliomas, maximal resection was not documented in many cases, adjuvant therapy was used in a non-protocol driven fashion, and follow-up was shorter. Although our series is small, it is the only prospective series of pure low-grade oligodendrogliomas managed with maximal resection and no adjuvant therapy, and with long term follow up available. Withholding adjuvant therapy at diagnosis appears to be safe. It will be important to establish the molecular differences between the patients who had no evidence of tumor progression and those who progressed so that adjuvant therapy could be offered to the latter.

\section{REFERENCES}

[1] S. Chowdhary and M. C. Chamberlain, "Oligodendroglial tumors," Expert Review of Neurotherapeutics, Vol. 6, No. 4, 2006, pp. 519-532. doi:10.1586/14737175.6.4.519

[2] B. Devaux, B. Turak, T. Roujeau, P. Page, C. Cioloca, A. C. Ricci, P. Bret, F. Nataf and F. X. Roux, "Adult Supratentorial Oligodendrogliomas. Surgical Treatment: Indications and Techniques," Neurochirurgie, Vol. 51, No. 3-4(Part 2), 2005, pp. 353-367.

[3] H. H. Engelhard, "Current Diagnosis and Treatment of Oligodendroglioma,” Neurosurg Focus, 2002, p. 12:E2.

[4] W. P. Mason, "Oligodendroglioma," Current Treatment Options in Neurology, Vol. 7, No. 4, 2005, pp. 305-314. doi:10.1007/s11940-005-0040-0

[5] G. Ozyigit, C. Onal, M. Gurkaynak, F. Soyiemezoglu and F. Zorlu, "Postoperative Radiotherapy and Chemotherapy in the Management of Oligodendroglioma: Single Institutional Review of 88 Patients," Journal of Neuro-Oncology, Vol. 75, No. 2, 2005, pp. 189-193. doi:10.1007/s11060-005-2057-3

[6] M. R. Rosenfeld, "Should Radiotherapy for Low-Grade Glioma be Given Immediately after Surgery or at the Time of Progression?” Nature Clinical Practice Neurology, Vol. 2, No. 3, 2006, pp. 128-129. doi:10.1038/ncpneuro0128

[7] F. X. Roux and F. Nataf, "Cerebral Oligodendrogliomas in Adults and Children. Current Data and Perspectives," Neurochirurgie, Vol. 51, No. 3-4(Pt 2), 2005, pp. 410-414.

[8] J. Piepmeier, S. Christopher, D. Spencer, T. Byrne, J. Kim, J. P. Knisel, J. Lacy, L. Tsukerman and R. Makuch, "Variations in the Natural History and Survival of Patients with Supratentorial Low-Grade Astrocytomas," Neurosurgery, Vol. 38, No. 5, 1996, pp. 872-878. doi:10.1097/00006123-199605000-00002

[9] S. Puget and J. T. Rutka, "Malignant Brain Tumors: Two Steps Forward," Clinical Neurosurg, Vol. 54, 2007, pp 4-9.

[10] P. Kleihues, D. N. Louis, B. W. Scheithauer, L. B. Rorke,
G. Reifenberger, P. C. Burger and W. K. Cavenee, "The WHO Classification of Tumors of the Nervous System," Journal of Neuropathology \& Experimental Neurology, Vol. 61, No. 3, 2002, pp. 215-225.

[11] M. J. van den Bent, "Advances in the Biology and Treatment of Oligodendrogliomas," Current Opinion in Neurology, Vol. 17, No. 6, 2004, pp. 675-680. doi:10.1097/00019052-200412000-00006

[12] C. Walker, D. G. du Plessis, K. A. Joyce, D. Fildes, A. Gee, B. Haylock, D. Husband, T. Smith, J. Broome and P. C. Warnke, "Molecular Pathology and Clinical Characteristics of Oligodendroglial Neoplasms," Annals of Neurology, Vol. 57, No. 6, 2005, pp. 855-865. doi:10.1002/ana.20496

[13] A. A. Brandes, A. Tosoni, G. Cavallo, M. Reni, E. Franceschi, L. Bonaldi, R. Bertorelle, M. Gardiman, C. Ghimenton, P. Iuzzolino, A. Pession, V. Blatt and M. Ermani, "Correlations between O6-Methylguanine DNA Methyltransferase Promoter Methylation Status, 1p and 19q Deletions, and Responses to Temozolomide in Anaplastic and Recurrent Oligodendroglioma: A Prospective GICNO Study,” Journal of Clinical Oncology, Vol. 24, No. 29, 2006, pp. 4746-4753. doi:10.1200/JCO.2006.06.3891

[14] J. G. Cairncross, K. Ueki, M. C. Zlatescu, D. K. Lisle, D. M. Finkelstein, R. R. Hammond, J. S. Silver, P. C. Stark, D. R. MacDonald, Y. Ino, D. A. Ramsay and D. N. Louis, "Specific Genetic Predictors of Chemotherapeutic Response and Survival in Patients with Anaplastic Oligodendrogliomas," Journal of the National Cancer Institute, Vol. 90, No. 19, 1998, pp. 1473-1479. doi:10.1093/jnci/90.19.1473

[15] K. A. Jaeckle, K. V. Baliman, R. D. Rao, R. B. Jenkins and J. C. Buckner, "Current Strategies in Treatment of Oligodendroglioma: Evolution of Molecular Signatures of Response,” Journal of Clinical Oncology, Vol. 24, No. 8, 2006, pp. 1246-1252. doi:10.1200/JCO.2005.04.9874

[16] R. B. Jenkins, H. Blair, K. V. Ballman, C. Giannini, R. M. Arusell, M. Law, H. Flynn, S. Passe, S. Felton, P. D. Brown, E. G. Shaw and J. C. Buckner, “A T[1;19][q10;p10] Mediates the Combined Deletions of $1 p$ and $19 q$ and Predicts a Better Prognosis of Patients with Oligodendroglioma," Cancer Research, Vol. 66, No. 20, 2006, pp. 9852-9861. doi:10.1158/0008-5472.CAN-06-1796

[17] N. Levin, I. Lavon, B. Zelikovitsh, D. Fuchs, F. Bokstein, Y. Fellig and T. Siegal, "Progressive Low-Grade Oligodendrogliomas: Response to Temozolomide and Correlation between Genetic Profile and O6-Methylguanine DNA Methyltransferase Protein Expression,” Cancer, Vol. 106, No. 8, 2006, pp. 1759-1765. doi:10.1002/cncr.21809

[18] G. E. Keles, K. R. Lamborn and M. S. Berger, "Low Grade Hemispheric Gliomas in Adults: A Critical Review of Extent of Resection as a Factor Influencing Outcome,” Journal of Neurosurg, Vol. 95, No. 5, 2001, pp. 735-745. doi:10.3171/jns.2001.95.5.0735 
[19] N. Pouratian, A. Asthagiri, J. Jagannathan, M. E. Shaffrey and D. Schiff, "Surgery Insight: The Role of Surgery in the Management of Low-Grade Gliomas," Nature Clinical Practice Neurology, Vol. 3, No. 11, 2007, pp 628-639. doi:10.1038/ncpneuro0634

[20] J. S. Smith, E. F. Chang, K. R. Lamborn, S. M. Chang, M. D. Prados, S. Cha, T. Tihan, S. Vandenberg, M. W. McDermott and M. S. Berger, "Role of Extent of Resection in the Long-Term Outcome of Low-Grade Hemispheric Gliomas,” Journal of Clinical Oncology, Vol. 26, No. 8, 2008, pp. 1338-1345. doi:10.1200/JCO.2007.13.9337

[21] C. Leighton, B. Fisher, G. Bauman, S. Depiero, L. Stitt, D. MacDonald and G. Cairncross, "Supratentorial Low Grade Glioma in Adults: An Analysis of Prognostic Factors and Timing of Radiation," Journal of Clinical Oncology, Vol. 15, No. 4, 1997, pp. 1294-1301.

[22] F. Pignatti, M. van den Bent, D. Curran, C. Debruyne, R. Sylvester, P. Therasse, D. Afra, P. Cornu, M. Bolla, C. Vecht and A. B. Karim, "European Organization for Research and Treatment of Cancer Brain Tumor Cooperative Group; European Organization for Research and Treatment of Cancer Radiotherapy Cooperative Group; Prognostic Factors for Survival in Adult Patients with Cerebral Low-Grade Glioma," Journal of Clinical Oncology, Vol. 20, No. 8, 2002, pp. 2076-2084. doi:10.1200/JCO.2002.08.121

[23] E. G. Shaw, S. B. Tatter, G. J. Lesser, T. L. Ellis, C. A. Stanton and V. W. Stieber, "Current Controversies in the Radiotherapeutic Management of Adult Low Grade Glioma,” Seminars in Oncology, Vol. 31, No. 5, 2004, pp. 653-658. doi:10.1053/j.seminoncol.2004.07.007

[24] S. E. Combs, D. Schulz-Ertner, C. Thilmann, L. Edler and J. Debus, "Fractionated Stereotactic Radiation Therapy in the Management of Primary Oligodendroglioma and Oligoastrocytoma," International Journal of Radiation Oncology, Vol. 62, No. 3, 2005, pp. 797-802. doi:10.1016/j.ijrobp.2004.11.011

[25] E. B. Claus, A. Horlacher, L. Hsu, R. B. Schwartz, D. Dello-Iacono, F. Talos, F. A. Jolesz and P. M. Black, "Survival Rates in Patients with Low-Grade Glioma after Intraoperative Magnetic Resonance Image Guidance," Cancer, Vol. 103, No. 6, 2005, pp. 1227-1233. doi:10.1002/cncr.20867

[26] M. S. Berger, A. V. Deliganis, J. Dobbins and G. E. Keles, "The Effect of Extent of Resection on Recurrence in Patients with Low Grade Cerebral Hemisphere Gliomas,” Cancer, Vol. 74, No. 6, 1994, pp. 1784-1791. doi:10.1002/1097-0142(19940915)74:6<1784::AID-CNC $\underline{\mathrm{R} 2820740622>3.0 . \mathrm{CO} ; 2-\mathrm{D}}$

[27] M. Nakamura, N. Konishi, S. Tsunoda, H. Nakase, T. Tsuzuki, H. Aoki, H. Sakitani, T. Inui and T. Sakaki, "Analysis of Prognostic and Survival Factors Related to Treatment of Low-Grade Astrocytomas in Adults," Oncology, Vol. 58, No. 2, 2000, pp. 108-116. doi:10.1159/000012087

[28] P. Celli, I. Nofrone, L. Palma, G. Cantore and A. Fortuna,
"Cerebral Oligodendroglioma: Prognostic Factors and Life History,” Neurosurgery, Vol. 35, No. 6, 1994, pp. 1018-1034. doi:10.1227/00006123-199412000-00003

[29] K. F. Lindegaard, S. J. Mork, G. E. Eide, T. B. Halvorsen, R. Hatlevoll, T. Solgaard, O. Dahl and J. Ganz, "Statistical Analysis of Clinico-Pathological Features, Radiotherapy, and Survival in 170 Cases of Oligodendroglioma,” Journal of Neurosurgery, Vol. 67, No. 2, 1987, pp. 224-230. doi:10.3171/jns.1987.67.2.0224

[30] E. G. Shaw, B. W. Scheithauer, J. R. O’Fallon, H. D. Tazelaar and D. H. Davis, "Oligodendrogiomas: The Mayo Clinic Experience,” Journal of Neurosurgery, Vol. 76, No. 3, 1992, pp. 428-434. doi:10.3171/jns.1992.76.3.0428

[31] S. A. Yeh, J. T. Ho, C. C. Lui, Y. J. Huang, C. Y. Hsiung and E. Y. Huang, "Treatment Outcomes and Prognostic Factors of Patients with Supratentorial Low-Grade Oligodendroglioma," International Journal of Radiation Oncology Biology Physics, Vol. 54, No. 5, 2002, pp. 1405-1409. doi:10.1016/S0360-3016(02)03053-5

[32] A. B. Karim, B. Maat, R. Hatlevoll, J. Menten, E. H. Rutten, D. G. Thomas, F. Mascarenhas, J. C. Horiot, L. M. Parvinen, M. van Reijn, J. J. Jager, M. G. Fabrini, A. M. van Alphen, H. P. Hamers, L. Gaspar, E. Noordman, M. Pierart and M. van Glabbeke, "A Randomized Trial on Dose-Response in Radiation Therapy of Low-Grade Cerebral Glioma: European Organization for Research and Treatment of Cancer [EORTC] Study 22844,” International Journal of Radiation Oncology, Vol. 36, No. 3 1996, pp. 549-556. doi:10.1016/S0360-3016(96)00352-5

[33] E. Shaw, R. Arusell, B. Scheithauer, J. O’Fallon, B. O’Neill, R. Dinapoli, D. Nelson, J. Earle, C. Jones, T. Cascino, D. Nichols, R. Ivnik, R. Hellman, W. Curran and R. Abrams, "Prospective Randomized Trial of LowVersus High-Dose Radiation Therapy in Adults with Supratentorial Low-Grade Glioma: Initial Report of a North Central Cancer Treatment Group/Radiation Therapy Oncology Group/ Eastern Cooperative Oncology Group study," Journal of Clinical Oncology, Vol. 20, No. 9, 2002, pp. 2267-2276. doi:10.1200/JCO.2002.09.126

[34] M. J. Van den Bent, D. Afra, O. de Witte, M. Ben Hassel, S. Schraub, K. Hoang-Xuan, P. O. Malmstrom, L. Collette, M. Pierart, R. Mirimanoff and A. B. Karim, "EORTC Radiotherapy and Brain Tumor Groups and the UK Medical Research Council, Long-Term Efficacy of Early Versus Delayed Radiotherapy for Low-Grade Astrocytoma and Oligodendroglioma in Adults: The EORTC 22845 Randomised Trial,” Lancet, Vol. 366, No. 9490, 2005, pp. 985-990. doi:10.1016/S0140-6736(05)67070-5

[35] J. Knisely, "Early or Delayed Radiotherapy for Low-Grade Glioma?” Lancet, Vol. 6, No. 12, 2005, p. 921.

[36] Y. Higuchi, Y. Iwadate and A. Yamamura, "Treatment of Low-Grade Oligodendroglial Tumors without Radiotherapy,” Neurology, Vol. 63, No. 12, 2004, pp. 2384-2386.

[37] O. Surma-aho, M. Niemela, J. Vijkki, M. Kouri, A. Brander, O. Salonen, A. Paetau, M. Kallio, J. Pyykkönen 
and J. Jääskeläinen, “Adverse Long-Term Effects of Brain Radiotherapy in Adult Low-Grade Glioma Patients,” Neurology, Vol. 56, No. 10, 2001, pp. 1285-1290.

[38] M. Klein, J. J. Heimans, N. K. Aaronson, P. M. van der Ploeg, J. Grit, M. Muller, T. J. Postma, J. J. Mooij, R. H. Boerman, G. N. Beute, G. J. Ossenkoppele, G. W. van Imhoff, A. W. Dekker, J. Jolles, B. J. Slotman, H. Struikmans and M. J. Taphoorn, "Effect of Radiotherapy and other Treatment-Related Factors on Mid-Term to Longterm Cognitive Sequelae in Low Grade Gliomas: A
Comparative Study,” Lancet, Vol. 360, No. 9343, 2002. pp. 1361-1368. doi:10.1016/S0140-6736(02)11398-5

[39] L. R. Rogers, H. H. Morris and K. Lupica, "Effect of Cranial Irradiation on Seizure Frequency in Adults with Low-Grade Astrocytoma and Medically Intractable Epilepsy,” Neurology, Vol. 43, No. 8, 1993, pp 1599-1601.

[40] J. D. Olson, E. Riedel and L. M. DeAngelis, "Long-Term Outcome of Low-Grade Oligodendroglioma and Mixed Glioma,” Neurology, Vol. 54, No. 7, 2000, pp. 1442 1448. 